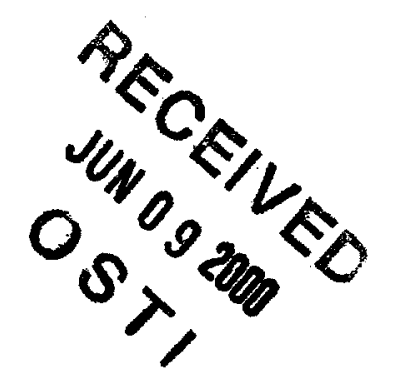

\title{
SIMULATIONS OF SURFACE WAVES GENERATED USING LASER ULTRASONICS
}

\author{
J. J. Dike \\ Solid \& Material Mechanics Department \\ Sandia National Laboratories \\ Livermore, CA 94551-0969
}

T. M. Sanderson

Georgia Institute of Technology

School of Mechanical Engineering

Atlanta, GA 30032

\section{INTRODUCTION}

Computer programs for solving the thermoelastic equations describing wave generation and propagation caused by the interaction of a laser pulse with a metal surface have been developed over the last several years [1-3]. One approach is to manipulate the thermoelastic equations using transform techniques and then use numerical methods to invert the equations and solve for wave displacements. Another approach is to spatially discretize the geometry of the model using finite elements and integrate the equations of motion thisough time. The finite element formulation may be fully coupled or as a further approximation the thermal problem can be solved separately from the mechanical problem. The work reported here sought to develop a technique to use a commercial finite element code (ABAQUS [4]) to simulate surface waves generated in laser ultrasonics. A general purpose finite element code provides the advantages of large element and material libraries and the ability to consider complex geometries and boundary conditions. Sanderson's [3] computer code, which solves the coupled thermoelastic problem using numerical transform techniques, was used to validate the finite element model developed. Validation was performed using simple models and boundary conditions. Subsequent finite element simulations were used to examine the effects of simulated stress gradients (in-plane and through-thickness) on waveforms. Temperature dependent properties and the effect of including an elastic-plastic constitutive material model in the mechanical analysis were also briefly examined.

A robust ability to predict waveforms occurring in laser ultrasonics would be of great general use. The capability could be used to help develop techniques for narrow band generation of laser 


\section{DISCLAIMER}

This report was prepared as an account of work sponsored by an agency of the United States Government. Neither the United States Government nor any agency thereof, nor any of their employees, make any warranty, express or implied, or assumes any legal liability or responsibility for the accuracy, completeness, or usefulness of any information, apparatus, product, or process disclosed, or represents that its use would not infringe privately owned rights. Reference herein to any specific commercial product, process, or service by trade name, trademark, manufacturer, or otherwise does not necessarily constitute or imply its endorsement, recommendation, or favoring by the United States Government or any agency thereof. The views and opinions of authors expressed herein do not necessarily state or reflect those of the United States Government or any agency thereof. 


\section{DISCLAIMER}

Portions of this document may be illegible in electronic image products. Images are produced from the best available original document. 
ultrasonic waves to provide control of wave frequency content. This would allow the depth of probing to be controlled which would be of use in evaluating layered media and media with through-thickness stress gradients. Model-based signal processing techniques [ie, Ref. 5] to improve signal to noise ratios and time-of-flight measurements, which would lead to more accurate measurements of stress and velocities, could be applied if accurate predictive methods are available.

\section{PROBLEM DESCRIPTION}

The physical situation considered here is shown in Figure 1. A laser pulse with known spatial and temporal distribution is applied to the surface of an aluminum plate. The laser pulse heats a volume of material around the point of application and a rapid thermal expansion occurs, generating stress and displacement waves. Here we focus on out-of-plane displacements observed on the same surface to which the laser is applied. Laser pulses simulated for the results presented here were Gaussian in space and time, with spot diameters from 2.25 to $4 \mathrm{~mm}$, and pulse duration of 4 ns. Most pulse energies were $0.2 \mathrm{~mJ}$.

\section{SIMULATIONS}

Two techniques were used to simulate laser generated surface waves. A semi-analytical approach developed and implemented into a computer code by Sanderson [3] was used to generate waveforms against which finite element model results were compared. Hankel-Laplace transforms were used in [3] to recast the equations of thermoelasticity. Once the equations have been developed for in-plane and out-of-plane dispacements in terms of the transform variables, numerical techniques for inverting the transformed equations were applied. In the second simulation technique, uncoupled thermal and mechanical finite element analyses were performed. Results from the two methods were compared for an elastic, stress free material, with temperature independent properties. As Sanderson's results were validated by experiments, good agreement between finite element and transform method results imply good agreement between finite element model results and experiments. Once good agreement was obtained for this ideal case, finite element models were extended to consider the effects of temperature dependent properties, an elastic-plastic mechanical constitutive model, and in-plane and out-of-plane stress gradients.

\section{Surface Waves from Solution of Hankel-Laplace Transformed Equations of Thermoelasticity}

The starting equations are those proposed by Lord and Schulman [6]. This formulation eliminates the infinite speed of the thermal wave predicted by classical thermoelasticity, and the results can easily be modified to solve the Green-Lindsay [7] and classical formulations. Details of

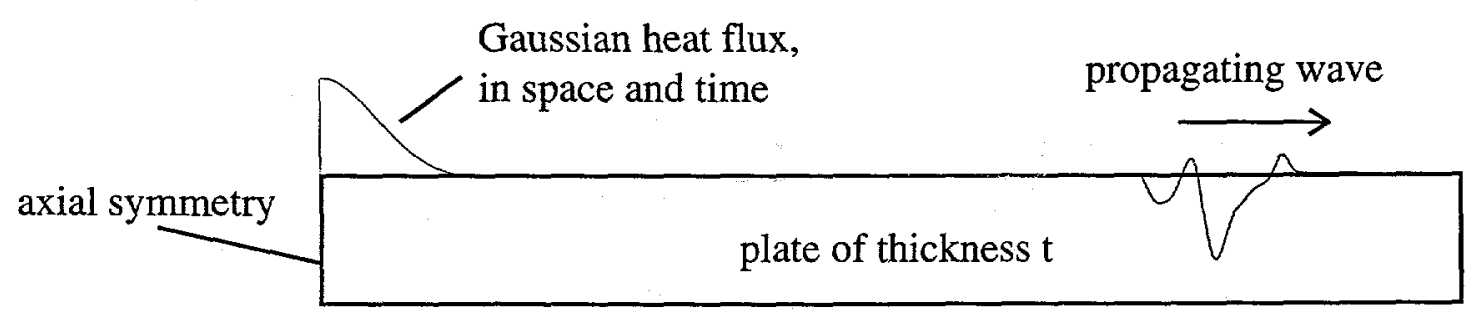

Figure 1. Schematic of surface wave generated by laser pulse. 
the formulation and the Hankel-Laplace transformations are provided in [3]. The resulting transformed expressions that must be evaluated are

$$
\begin{aligned}
& \mathrm{u}_{\mathrm{r}}(\mathrm{r}, \mathrm{z}, \mathrm{t})=\frac{1}{2 \pi \mathrm{i}} \int_{0}^{\infty} \int_{\alpha_{\mathrm{p}}-\mathrm{i} \infty}^{\alpha_{\mathrm{p}}+i \infty} \gamma \mathrm{J}_{1}(\gamma \mathrm{r}) \mathrm{e}^{s t} \tilde{\overline{\mathrm{u}}}_{\mathrm{r}}(\gamma ; \mathrm{z} ; \mathrm{s}) \mathrm{ds} \mathrm{d} \gamma \\
& \mathrm{w}(\mathrm{r}, \mathrm{z}, \mathrm{t})=\frac{1}{2 \pi \mathrm{i}} \int_{0}^{\infty} \int_{\alpha_{\mathrm{p}}-\mathrm{i} \infty}^{\alpha_{\mathrm{p}}+i_{\infty}} \gamma \mathrm{J}_{\mathrm{o}}(\gamma \mathrm{r}) \mathrm{e}^{s t} \tilde{\overline{\mathrm{w}}}(\gamma ; \mathrm{z} ; \mathrm{s}) \mathrm{dsd} \gamma
\end{aligned}
$$

where $u_{r}$ and $w$ are the radial and out-of-plane (z-direction) displacements, respectively, $J_{0}$ and $J_{1}$ are ordinary Bessel functions, and $\gamma$ and $s$ are the Hankel and Laplace transform variables, respectively. The variables $\widetilde{\overline{\mathrm{u}}}_{\mathrm{r}}$ and $\widetilde{\mathrm{w}}$ are the Hankel-Laplace transformed displacements. The inverse Hankel transform is an infinite range oscillatory integral while the inverse Laplace transform is an infinite range line integral in the complex plane. Crump's [8] numerical Laplace inversion formula was implemented to solve the inner integral. Clenshaw-Curtis [9] quadrature was used to solve the outer integral. The resulting expression evaluated for the radial displacement component is s:

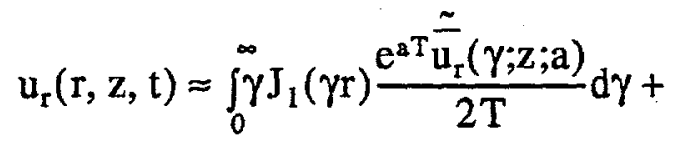

$$
\begin{aligned}
& \frac{\mathrm{e}^{\mathrm{aT}}}{\mathrm{T}} \sum_{\mathrm{k}=1}^{\infty}\left\{\int_{0}^{\infty} \gamma \mathrm{J}_{1}(\gamma \mathrm{r}) \operatorname{Re}\left[\frac{\tilde{-}}{\mathrm{u}_{\mathrm{r}}}\left(\gamma ; \mathrm{z} ; \mathrm{a}+\mathrm{i} \frac{\mathrm{k} \pi}{\mathrm{T}}\right)\right] \cos \left(\frac{\mathrm{k} \pi \mathrm{t}}{\mathrm{T}}\right) \mathrm{d} \gamma\right. \\
& \left.-\int_{0}^{\infty} \gamma \mathrm{J}_{1}(\gamma \mathrm{r}) \operatorname{Im}\left[\frac{\tilde{\mathrm{u}}}{\mathrm{r}}\left(\gamma ; \mathrm{z} ; \mathrm{a}+\mathrm{i} \frac{\mathrm{k} \pi}{\mathrm{T}}\right)\right] \sin \left(\frac{\mathrm{k} \pi \mathrm{t}}{\mathrm{T}}\right) \mathrm{d} \gamma\right\} \text {. }
\end{aligned}
$$

A similar expression is obtained for the out-of-plane displacement, $w$.

Some assumptions made in the development of Equation (3) are that material properties are constant and the material is linearly elastic. Another aspect of solutions generated analytically or through transform techniques is that the solution is typically provided at a single point per execution of the code. Observation of the response over the body is not typically feasible. These solution techniques also are limited by the assumption simple geometries and boundary conditions. It is recognized, however, that in many cases the response at a single point on a flat stress-free surface in a linearly elastic, temperature independent material is all that is being sought. This makes analytical and transform technique solutions extremely valuable by themselves as well as for validating other types of analyses.

\section{Finite Element Simulations of Surface Waves Generated by a Laser Pulse}

Finite element models, in which the structure's geometry is discretized and the weak form of the governing equations are satisfied over the body, provide the capability to overcome many of the limitations imposed on analytical and semi-analytical solution techniques. General material behavior is easily accomodated, as well as complex geometries and boundary conditions. These models are also capable of providing results over the entire part over time, allowing a better understanding of the analysis. However, due to the small wavelengths of waves generated in laser 
ultrasonics, the structure must be finely discretized spatially. If the response due to the laser pulse must be available over an area large compared to the wavelengths of interest, a large number of finite elements can be required. In this section a finite element model is presented and solutions obtained using the commercial finite element code ABAQUS [4] are discussed. The assumption in the finite element model is that the thermal and mechanical analyses may be uncoupled. When the time scale of interest is long relative to the duration of the pulse, this uncoupling is reasonable. Displacements caused by the laser pulse are only on the order of nanometers, and so will not significantly influence the diffusion or deposition of energy over the few microseconds of a waveform duration.

\section{Thermal Finite Element Analyses}

Many of the assumptions regarding the thermal response used in the transform solutions are also present in the finite element models. As discussed in [3], the thermal expansion that occurs due to laser heating occurs over a time span close to that of the pulse duration ( $4 \mathrm{~ns}$ here). Subsequent diffusion of the heat from the surface takes place on a time scale typically much larger than that of the wave duration. This, in addition to the very small displacements that arise (order of $\mathrm{nm}$ ), allows the interaction of the material displacement with the diffusion of thermal energy to be neglected. As was assumed in the transform technique solutions [3], thermal losses due to radiation and convection are ignored. Over the time scale of interest these losses are an order of magnitude smaller than the energy absorbed in the specimen $[1,10]$. The heat source is modeled as a flux on the surface, rather than a volume source, because the depth of penetration is very small during the time of wave generation. For a $4 \mathrm{~ns}$ pulse with $2 \mathrm{~mm}$ spot diameter, energy diffuses only to about 1 $\mu \mathrm{m}$ in aluminum by $4 \mathrm{~ns}$, approximately $7 \mu \mathrm{m}$ by $100 \mathrm{~ns}$, and $70 \mu \mathrm{m}$ by $6 \mu \mathrm{s}$. An analysis by $\mathrm{McDonald}$ [11] showed that for metals the heat deposition is well modeled by a surface flux for most applications.

The thermal finite element models consisted of a line of axisymmetric shell elements (ABAQUS type DSAX1), which are 2 node diffusion elements. A Gaussian surface flux of $4 \mathrm{~ns}$ duration and $0.2 \mathrm{~mJ}$ energy was applied in a Gaussian distribution over spot radii of 1.125 and 2 $\mathrm{mm}$ using the ABAQUS user subroutine DFLUX. Three element lengths were examined, 0.025 $\mathrm{mm}, 0.05 \mathrm{~mm}$, and $0.1 \mathrm{~mm}$ which matched element sizes in the mechanical analyses. Shell elements were $0.1 \mathrm{~mm}$ thick. Thermal properties used were thermal conductivity of $0.177 \mathrm{~W} / \mathrm{mm}^{2}$, density of $2.77 \times 10^{-3} \mathrm{~g} / \mathrm{mm}^{3}$, and a specific heat of $0.875 \mathrm{~J} / \mathrm{g}-\mathrm{K}$. The three element sizes considered gave essentially the same thermal profiles. The peak temperature change observed was approximately $325^{\circ} \mathrm{C}$, while the temperature at the bottom integration point $(0.1 \mathrm{~mm}$ deep $)$ increased by less than $1{ }^{\circ} \mathrm{C}$ at $10 \mu \mathrm{s}$.

\section{Mechanical Finite Element Analyses}

Meshes were constructed of 2-node linear axisymmetric shell elements (ABAQUS type SAX1) and square axisymmetric 4-node bilinear quadrilateral elements with reduced integration (ABAQUS type CAX4R). Shell elements were at the same location and had the same thickness as in the thermal analysis. Shell elements with 19 integration points through the thickness were again used. Solid element nodes coincident with shell element nodes were constrained to follow them through use of the TIED contact constraint in ABAQUS. In both thermal and mechanical analyses presented here, shell element meshes only extended to a radius of $4 \mathrm{~mm}$, which was larger than all laser spot radii considered. Mechanical meshes extended to $20 \mathrm{~mm}$ to avoid reflections from the edge of the model during the period of interest. ABAQUS also has an infinite element for modeling 
non-reflective boundaries that could be used to reduce the mesh size. Out of plane displacements were recorded $8 \mathrm{~mm}$ from the center of laser heating. Figure 2 shows a schematic of the mesh.

Element sizes of $0.1,0.05$, and $0.025 \mathrm{~mm}$ (associated with coarse, medium, and fine meshes, respectively) were selected as being able to capture Rayleigh waves of wavelengths 3,6 , and $12 \mathrm{MHz}$ respectively, assuming adequate resolution at 10 elements per wavelength and assuming a Rayleigh wave speed in aluminum of $3 \mathrm{~mm} / \mu \mathrm{s}$. Mechanical properties used were Young's modulus $=69 \mathrm{GPa}$, Poisson's ratio $=0.327$, and density of $2.77 \times 10^{-3} \mathrm{~g} / \mathrm{mm}^{3}$.

Results

Figure 3 shows a comparison of finite element results with transform technique results for 4 $\mathrm{mm}$ and $2.25 \mathrm{~mm}$ diameter spot sizes, $4 \mathrm{~ns}$ pulse duration, and $5 \mathrm{~mm}$ thick aluminum plate. Due to the similarity in waveforms for the medium and fine meshes, only the coarse and fine mesh results are shown. For both excitation diameters the coarse mesh underpredicts the peak associated with the Rayleigh wave arrival $(-2.7 \mu \mathrm{s})$ and the peak near $3.1 \mu \mathrm{s}$. The peak near $3.1 \mu \mathrm{s}$ occurs between the arrival of the Rayleigh wave from the epicenter and the arrival of the SV wave from the laser source far edge. Fine mesh magnitudes are slightly larger than those from the medium mesh. There is reasonably good agreement between the transform technique solution and the medium and fine finite element meshes. In Figure $3 b$, the fine and medium finite element method (FEM) solutions slightly overshoot the dip occuring near $2 \mu \mathrm{s}$, which is around the time the reflected $P$ wave and the

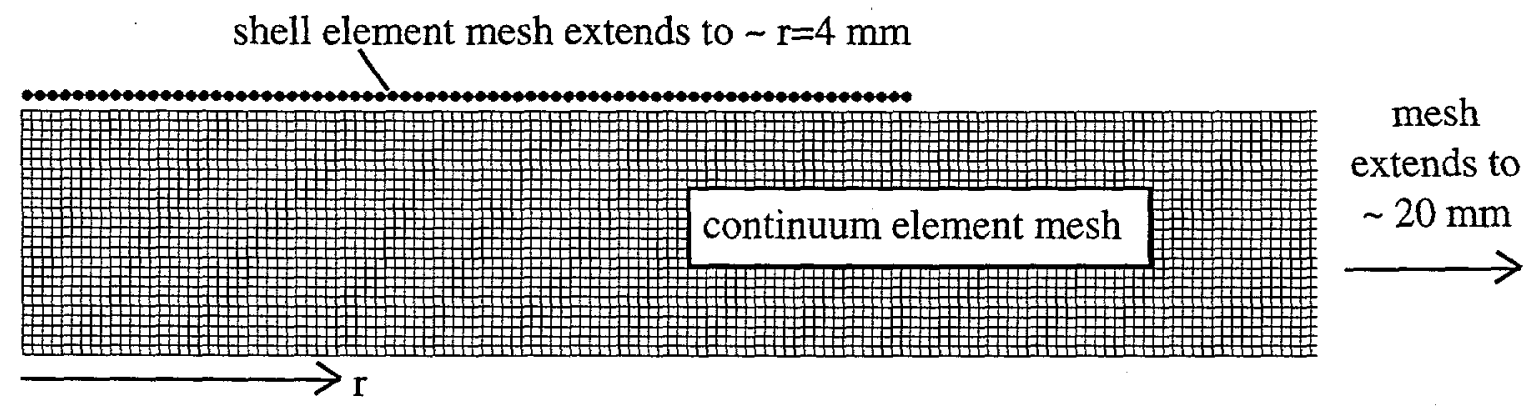

Figure 2. Schematic of mesh used in mechanical finite element analyses.
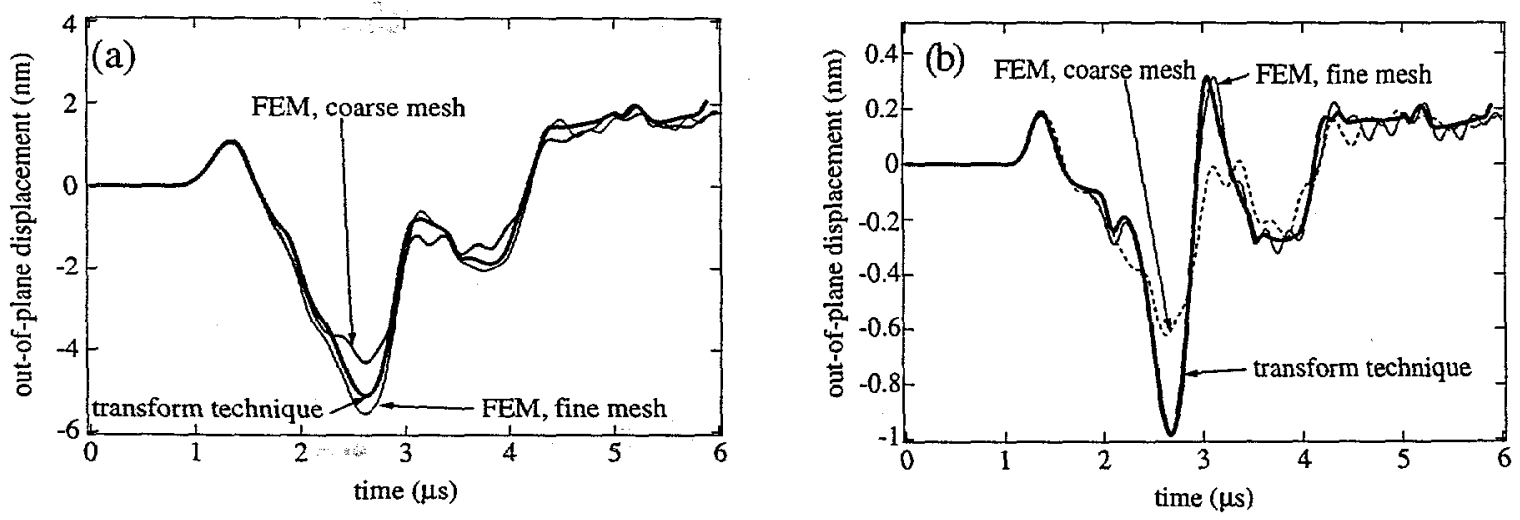

Figure 3. Comparison of finite element results for three mesh densities with results from transform solution. (a) Laser spot diameter of $4 \mathrm{~mm}$,(b) spot diameter of $2.25 \mathrm{~mm}$. Both plots are for $4 \mathrm{~ns}$ pulse duration and $5 \mathrm{~mm}$ thick aluminum plate. 
Rayleigh wave from the source near edge arrive. The peaks near $3.1 \mu$ s arrive slightly late in the FEM waveforms, and there is some oscillation in the FEM results after about $3 \mu \mathrm{s}$. Stiffness proportional damping was specified to minimize relatively high frequency ringing $(\sim 30 \mathrm{MHz})$ in the waveforms. The amount of damping added was typically on the order of $0.75 \mathrm{~ns}$ and was kept to the minimum required to prevent spurious oscillations as the addition of damping reduces the time step used in the analysis. Although agreement is good in a general sense and was adequate for purposes considered here, better agreement over the entire time of interest should be attainable. The temporal distributions of the heat flux are slightly different between the transform technique and finite element models, but those differences are not expected to significantly alter the waveforms.

\section{$\underline{\text { Simulated Stress Gradients }}$}

Once the waveforms for the case of temperature independent material properties, elastic mechanical response, and stress-free structure were determined to agree reasonably well, the finite element models were modified to briefly consider situations difficult if not impossible to solve with transform techniques. Figures $4 \mathrm{a}$ and $4 \mathrm{~b}$ show how waveforms change in the presence of simulated through-thickness and in-plane stress gradients, respectively. These waveforms were generated using the coarse finite element mesh to demonstrate the trends that might be expected. For the through-thickness case, stress gradients were simulated by varying Young's modulus in a way such that the wave speed varied linearly from its baseline value at the surface to a value of $10 \%$ or $20 \%$ greater at the bottom of the plate's $5 \mathrm{~mm}$ thickness. Similarly, the in-plane stress gradient case is simulated by modifying Young's modulus such that the wave speed varies linearly from its baseline value at $\mathrm{r}=0$ to a value $10 \%$ or $20 \%$ greater at the monitored location $\mathrm{r}=8 \mathrm{~mm}$. It is recognized that this method of simulating stress gradients is not totally correct but the general trends in the waveforms should be representative. One problem with simulating a stress gradient in this way is that the material is still isotropic. A more correct simulation is to treat the isotropic body with a prestress as an orthotropic body. The orthotropic elastic constants are modified from the elastic values by the amount of stress in the layer using relations developed by Man [11]. The stressed plate could be modeled by layers of orthotropic material with elastic constants modified by the amount of stress in each layer.

Figure 4a shows that as the magnitude of the stress gradient (wave speed) increases from its reference value at the surface to a greater value at the plate bottom, the longitudinal arrival time (around $1.2 \mu \mathrm{s}$ ) does not change significantly. This is observed because this part of the waveform is confined to the surface layer which has the baseline wave speed. However the Rayleigh wave arrival near $2.7 \mu$ s is reduced in magnitude and arrives sooner. Reflected waves which have traveled through the plate thickness also arrive sooner. The largest positive peak near $3 \mu$ s arrives earlier but does not undergo quite the same trend in magnitude change as the Rayleigh waves. Features of the waveform near $4 \mu$ s on the baseline curve have a fairly uniform shift in time and a still different trend in change in magnitude. For comparison, a waveform generated using an elastic modulus that gives a $20 \%$ higher wave speed throughout the body is also shown. Arrival times are uniformly $20 \%$ higher than that of the baseline case (no gradient), as expected, and no significant change in peak magnitudes is observed. Figure $4 b$ shows the $P$ wave arrival times decreasing as the simulated stress gradient is increased. The magnitudes of the P-waves $(\sim 1.2 \mu \mathrm{s})$ can be seen to be decreasing somewhat as the gradient is increased. Rayleigh waves, which travel along the free surface and whose penetration is frequency dependent, will experience the same increase in phase velocity at all frequencies because the in-plane stress gradient is uniform through the thickness. This is in contrast to the through-thickness gradient case (a), where Rayleigh waves with longer wavelengths will 
travel faster than those with shorter wavelengths since the wave speed is higher at the bottom of the plate.

Although the method for simulating the gradients is only approximate, the trends in waveforms are expected to be representative. More detailed explanations of the observed trends should be possible by studying the frequency and phase content of the waveforms.

\section{The Effect of Temperature Dependent Properties and Mechanical Constitutive Model}

A brief study was performed to examine the effect of using temperature dependent thermal and elastic material properties and to observe the effect of including plasticity in the mechanical model. Several combinations of temperature dependence in the thermal and mechanical analyses along with the inclusion of plasticity were considered. Four of the results are shown in Figure 5. In order for temperature dependence to play a more significant role, the energy of the laser pulse was increased from $0.2 \mathrm{~mJ}$ to $0.6 \mathrm{~mJ}$. This caused the highest temperature reached for the $r=1.125 \mathrm{~mm}$, $4 \mathrm{~ns}$ duration pulse to be close to the liquidus temperature for 6061-T6 aluminum (around $925 \mathrm{~K}$ ) when temperature dependent properties were used. When temperature independent thermal properties were used, the peak temperature was near $1360 \mathrm{~K}$. A bilinear elastic plastic constitutive model was used with a yield stress of $330 \mathrm{MPa}$ at room temperature decreasing linearly to approximately zero at the liquidus temperature. Forces due to ablation were not considered.

Figure 5 shows the results, along with a table showing the status of thermal and mechanical temperature dependence and if plasticity was considered. Using temperature dependent elastic and thermal properties significantly decreased the peak magnitudes in the waveform. The shape of the waveform was more affected by including plasticity.

\section{SUMMARY AND CONCLUSIONS}

A computer code [3] developed to solve the equations of thermoelasticity applied to laser ultrasonics was used to validate a finite element modeling technique for simulating surface waves generated by laser ultrasonics. The finite element model was then extended to briefly consider simulating stress gradients and the effects of temperature dependent properties and inelastic me
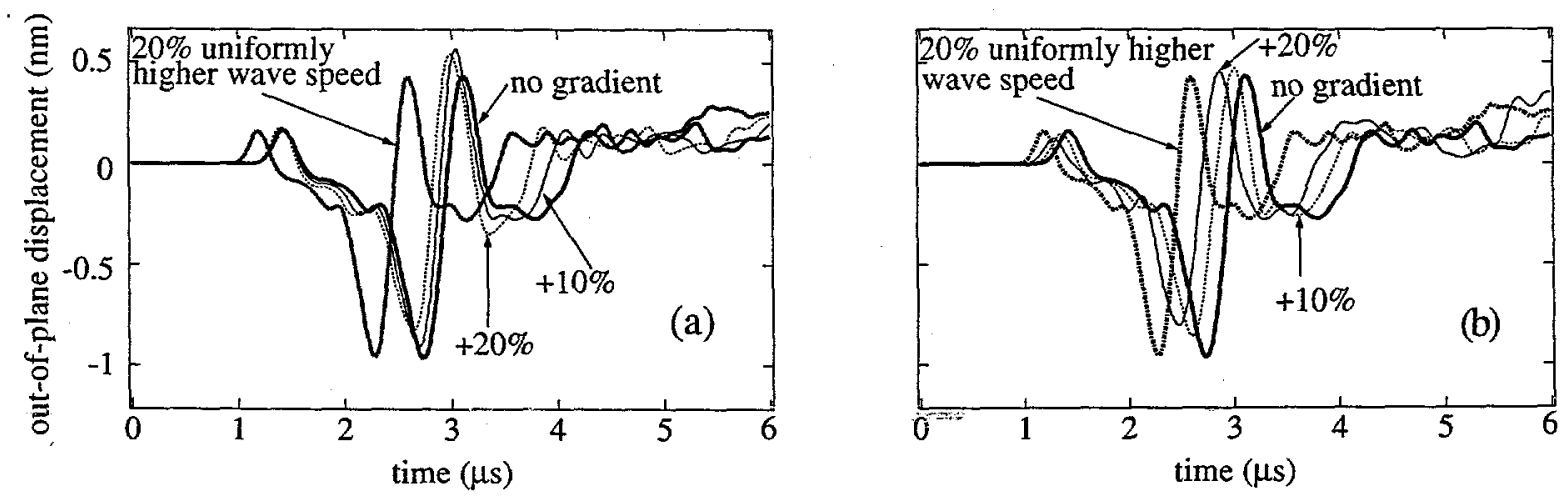

Figure 4. Effects of of (a) through-thickness, and (b) in-plane stress gradients simulated by varying Young's modulus. The $10 \%$ and $20 \%$ numbers reflect the increase in wave speed at the (a) plate bottom and (b) $8 \mathrm{~mm}$ radius relative to the unstressed wave speed. 


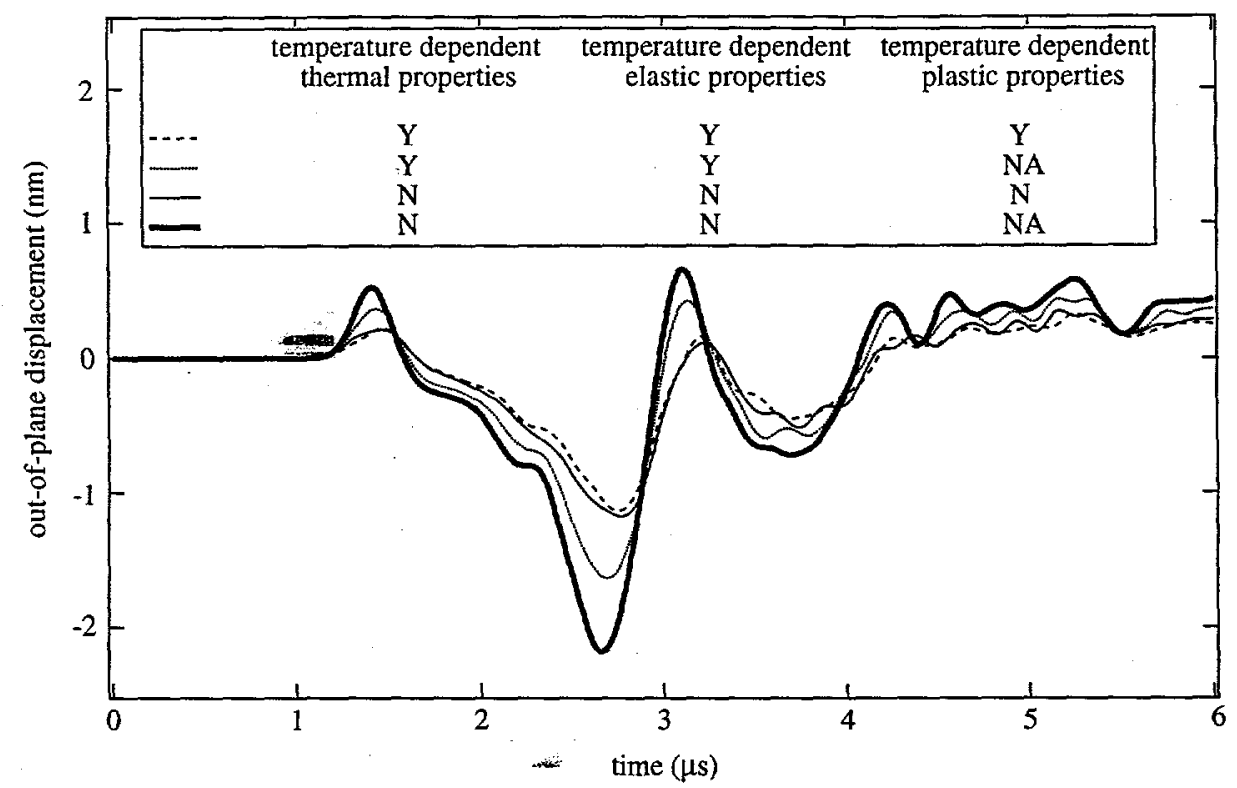

Figure 5. Finite element model waveforms showing effect of temperature dependent material properties and use of bilinear mechanical constitutive model. NA $=$ not applicable.

chanical constitutive models. Future work will focus on analyzing waveforms from this type of analysis and validating against experimental data, as well comparing with transform technique solutions for additional laser source diameters and plate thicknesses.

\section{ACKNOWLEDGEMENTS}

This work is supported by the U.S. Department of Energy, Sandia National Laboratories under contract DE-AC04-94AL85000 and is part of the Sandia Laboratory Directed Research and Development (LDRD) program.

\section{REFERENCES}

1. J. B. Spicer, "Laser Ultrasonics in Finite Structures: Comprehensive Modeling with Supporting Experiment", Ph.D. Thesis, The fôTins Hopkins University, 1991.

2. C. D. Suh, "Numerical Modeling of Laser Acoustic Waves using Finite Element Method and Fast Integral Wavelet Transform", Ph.D. Thesis, Texas A\&M University, 1997.

3. T. M. Sanderson, "Thermoelastic Modeling of Laser-Generated Ultrasound for Nondestructive Materials Testing", Ph.D. Thesis, Georgia Institute of Technology, 1997.

4. ABAQUS/Explicit and ABAQUS/Standard User's Manuals, Version 5.6, Hibbitt, Karlsson \& Sorensen, Inc.

5. J. V. Candy, G. H. Thomas, D. Chinn, and J. B. Spicer, "Laser Ultrasonic Signal Processing: A Model Reference Approach", Lawrence Livermore National Laboratory Report UCRL-JC-122414, Oct. 1995.

6. H. W. Lord and Y. Schulman, J. Mech. Phys. Solids, 15, p. 299 (1967).

7. A. E. Green and K. A. Linedsay, J. Elasticity, 2, p. 1 (1972).

8. K. S. Crump, J. Assoc. Comp. Mach., 23:1, p. 89 (1976).

9. C. W. Clenshaw and A. R. Curtis, Numer. Math., 7, p. 197 (1960).

10. C. B. Scruby and L. E. Drain, Laser Ultrasonics, Adam Hilger (1990).

11. A. F. McDonald, Appl. Phys. Letters, 56:3, p. 230 (1990).

12. C. S. Man, Inv. Prob., 14, p. 313 (1998). 